CLINICAL STUDY

\title{
Single photon emission computed tomography (SPECT)/computed tomography using Iodine-123 in patients with differentiated thyroid cancer: additional value over whole body planar imaging and SPECT
}

\author{
Tara Barwick, Iain Murray, Hakim Megadmi, William M Drake ${ }^{1}$, P Nick Plowman ${ }^{2}$, Scott A Akker ${ }^{1}$, \\ Shern L Chew ${ }^{1}$, Ashley B Grossman ${ }^{1}$ and Norbert Avril \\ Department of Nuclear Medicine, ${ }^{1}$ Department of Endocrinology and ${ }^{2}$ Department of Clinical Oncology, Barts and The London School of Medicine and NHS \\ Trust, Queen Mary, University of London, West Smithfield (QE II), London EC1A 7BE, UK \\ (Correspondence should be addressed to N Avril; Email: n.e.avril@qmul.ac.uk)
}

T Barwick is now at Department of Nuclear Medicine, Imperial College Healthcare NHS Trust, London, UK

\begin{abstract}
Objective: The aim of the study was to assess the diagnostic performance of co-registered single photon emission computed tomography (SPECT)/computed tomography (CT) compared to Iodine-123 whole body gamma camera (WBGC) imaging and to SPECT alone in patients with differentiated thyroid cancer.

Methods: WBGC and SPECT/CT $(n=85)$ imaging of the neck and thorax was performed in 79 consecutive patients. Three experienced observers reviewed: i) WBGC images followed by ii) SPECT alone, and iii) co-registered SPECT/CT. Foci of increased radioiodine uptake were classified on a fivepoint scale. Biopsy, other imaging modalities, and clinical follow-up served as the reference standard. Results: Twenty-two patients had local recurrence or metastatic thyroid cancer (11 were radioiodine negative), 9 had remnant thyroid tissue, and 54 had no evidence of disease. When classifying equivocal, probably, and definitely malignant findings as positive for malignancy, the sensitivity, specificity, positive predictive value, and negative predictive value were as follows: $41,68,31$, and $77 \%$ for WBGC imaging; 45, 89, 59, and 82\% for WBGC plus SPECT imaging; and 50, 100, 100, and 85\% for WBGC plus SPECT/CT imaging respectively. The specificity was improved by the addition of SPECT $(P=0.0002)$ and SPECT/CT $(P<0.0001)$ than to WBGC imaging. SPECT/CT was also more specific than WBGC plus SPECT imaging $(P=0.016)$. In a study-based analysis, SPECT/CT provided additional diagnostic information in $42 \%(36 / 85)$ of cases. SPECT/CT provided further characterization in $70 \%$ (63/90) of foci and improved the diagnostic confidence of all three observers.

Conclusion: The addition of SPECT/CT significantly improved the diagnostic information over Iodine123 WBGC imaging and WBGC plus SPECT imaging alone.

European Journal of Endocrinology 162 1131-1139
\end{abstract}

\section{Introduction}

Patients with differentiated thyroid cancer have an overall good prognosis; however, lifelong follow-up is required for many cases, since potentially curable local recurrences and distant metastases may occur even decades later (1-4). Radioiodine imaging plays a major role in the follow-up after initial surgery and ablation of residual thyroid tissue using Iodine-131 therapy, although the need for such extensive therapy for microcarcinomas has been questioned. Iodine-123 has replaced Iodine-131 at many institutions as a radionuclide for gamma camera imaging due to its more suitable physical characteristics (5).
However, the lack of anatomical details on planar gamma camera imaging and superimposition of areas presenting with increased radioiodine uptake can make accurate diagnosis and localization of radioiodine-avid metastatic disease challenging. This is particularly important in the neck and thorax where differentiation between physiological radioiodine accumulation, remnant thyroid tissue and residual or recurrent thyroid cancer may be difficult. Furthermore, accurate characterization and localization of thoracic radioiodine uptake to lung, bone, or lymph nodes can be equally challenging. In addition, altered anatomy, particularly in the neck and sites of physiological radioiodine accumulation such as in the esophagus, may be 
misinterpreted as metastatic disease (6, 7). For example, a retrospective review of 500 radioiodine whole body scans revealed diagnostic pitfalls leading to additional imaging or diagnostic procedures in $59 \%$ of studies (8).

Single photon emission computed tomography (SPECT) provides a 3D set of gamma camera images which improve the localization of increased tracer uptake within the body. This allows one to analyze the radioiodine distribution within the body in any plane; e.g. axial, coronal, and sagittal. However, precise anatomical localization is frequently still difficult due to a lack of appropriate landmarks on SPECT images. Integrated SPECT/computed tomography (CT) enables co-registration of structural and functional data and provides patient-specific data for the correction of photon attenuation $(9,10)$. This often permits improved spatial localization and characterization of increased tracer uptake which should ultimately lead to improved diagnostic performance in radioiodine imaging as well.

Most studies of SPECT/CT in thyroid cancer have used Iodine-131 to further evaluate equivocal findings on planar imaging. In our study, Iodine-123 SPECT/CT of the neck and thorax was performed in consecutive patients regardless of the findings on planar imaging. At our institution, thyroid cancer patients generally undergo thyroidectomy and radioiodine ablation, followed by endogenous TSH-stimulated Iodine-123 whole body planar gamma camera imaging and serum thyroglobulin measurement in high-risk patients 6 months later. Iodine-123 gamma camera imaging is also performed in patients with rising thyroglobulin levels. The routine protocol includes whole body planar imaging and SPECT/CT of the neck and thorax at $24 \mathrm{~h}$ after radioiodine injection.

This study represents a detailed analysis of the incremental value of the addition of Iodine-123 SPECT/CT compared with whole body planar imaging and SPECT alone. In addition, we assessed potential changes in patient management.

\section{Materials and methods}

\section{Patients}

Consecutive patients with differentiated thyroid cancer, who underwent Iodine-123 imaging from March 2006 to February 2009, were eligible for the study. Iodine123 gamma camera imaging was performed in patients with rising thyroglobulin levels as well as in high-risk patients 6 months following thyroidectomy and radioiodine ablation. Measurement of urinary iodine excretion was not performed prior to Iodine-123 administration. All the patients were imaged following endogenous TSH stimulation.

This retrospective analysis of patient data was performed as part of an audit, and institutional audit committee approval was obtained. The demographical and follow-up data were collected through a combination of review of clinic/correspondence letters/multidisciplinary meeting summaries and biochemical/histopathology databases on the electronic patient record, review of patient notes and direct communication with the physician in charge of the patient's care. The information for each patient was recorded on a patient case record form.

\section{Gamma camera and SPECT/CT imaging}

Whole body planar gamma camera (WBGC) images were acquired in the anterior and posterior projections $\sim 24 \mathrm{~h}$ post radiotracer injection $(350-400 \mathrm{MBq}$ Iodine-123 sodium iodide) on a dual-headed gamma camera (Millennium VG Hawkeye, GE Healthcare, Amersham, UK) with low energy general purpose collimators. The bed speed was $5 \mathrm{~cm} / \mathrm{min}$, and the data were acquired into a $256 \times 1024$ matrix, with a pixel size of $2.2 \mathrm{~mm}$. SPECT imaging of the neck and thorax was performed following WBGC imaging with $60 \mathrm{~s}$ frame time, step and shoot mode 360/60 frames ( $6^{\circ}$ angle step), 1.28 zoom. One patient also underwent an additional SPECT/CT of the abdomen and pelvis. SPECT data were reconstructed using an OSEM iterative reconstruction algorithm (Hermes, Nuclear Diagnostics Medical Systems, Sweden) into a $128 \times 128$ matrix, with a pixel size of $3.45 \mathrm{~mm}$ that incorporated the attenuation map information described below.

The CT acquisition was carried out using a fixed protocol $(140 \mathrm{kV}, 2.5 \mathrm{~mA})$ resulting in forty $10 \mathrm{~mm}$ slices that were subsequently rebinned to produce $1283.45 \mathrm{~mm}$ slices with a transverse pixel size of $1.7 \mathrm{~mm}$. The CT data were also rescaled to produce an attenuation map related to attenuation coefficient values at $159 \mathrm{keV}$ matching the dimensions of the reconstructed SPECT slices.

\section{Image analysis}

Gamma camera images were reviewed by three experienced observers ( $\mathrm{T} \mathrm{B}, \mathrm{H} \mathrm{M}, \mathrm{N}$ A) in a consensus reading without knowledge of clinical data on a dedicated workstation (Hermes Medical Solutions, Nuclear Diagnostics, Stockholm, Sweden) using a linear gray scale. For analysis of SPECT/CT images, both linear gray and color scale displays were used. The three clinicians reviewed the scans first separately followed by a consensus reading. In all cases, a consensus was reached.

The whole body planar images were reviewed initially followed by the SPECT and then the SPECT/CT. For Iodine-123 whole body planar imaging, symmetrical uptake in the salivary glands, linear uptake in the region of the esophagus, and uptake in the stomach, gastrointestinal tract and bladder were considered normal. Any focal increased uptake outside these areas was considered abnormal. Radioiodine uptake in the neck which was localized in the area of the thyroid 
bed was considered residual thyroid tissue following previous radioiodine ablation.

Sites of increased tracer uptake were noted along with the likelihood of the presence of disease according to the following five-point grading scale for whole body planar images, SPECT alone, and SPECT/CT:

\section{i) definitely normal}

ii) probably normal (more likely to be physiological)

iii) equivocal (equally likely to be pathological or physiological)

iv) probably abnormal (more likely to be pathological)

v) definitely abnormal.

For SPECT and SPECT/CT, the anatomical localization of increased radioiodine uptake was taken into account when reviewing images. Although we used a low-dose CT for anatomical correlation, the lung and bone windows were also reviewed for lung nodules and bone metastases. In addition, the overall diagnostic confidence score for each patient was allocated using the same five-point scale. For subsequent analysis, definitely abnormal, probably abnormal, and equivocal findings were considered malignant. Probably normal and definitely normal findings were considered benign. Thyroglobulin levels or findings from neck ultrasound, or any other clinical information were not made available to the image reviewers.

\section{Statistical analysis}

The sensitivity, specificity, positive predictive value, and negative predictive value were calculated for whole body planar gamma camera imaging, SPECT, and SPECT/CT on a study basis. For the purpose of these calculations, equivocal findings were considered malignant. Comparison of the detection of thyroid cancer metastases by each method was performed using an exact McNemar's test with a two-tailed $P$ value $<0.05$ being statistically significant (11). Following the exclusion of 11 studies found on follow-up to have non-radioiodineavid disease, receiver operating characteristic (ROC) curve analysis was performed by recalculating sensitivity and specificity for each technique along the fivepoint grading scale. In addition, the area under the curve (AUC) was calculated.

\section{Results}

Ninety-three patients underwent Iodine-123 imaging for follow-up of thyroid cancer from March 2006 to February 2009; however, in 12 cases SPECT/CT was not performed due to logistical and/or technical reasons. In one case, neck movement resulted in substantial image misregistration, and one patient with normal planar and SPECT/CT imaging was lost to follow-up. Therefore, the study population consisted of 79 patients in whom a total of 85 Iodine-123 imaging studies were performed. Patient characteristics are shown in Table 1.

Of these cases, 64 studies were performed as routine follow-up of high-risk patients 6 months after radioiodine ablation of residual thyroid tissue; five studies were performed 6 months after radioiodine therapy for completion of ablation $(n=3)$ or for metastatic disease. Twelve studies were performed in patients with rising or elevated thyroglobulin. The remaining three cases were to exclude recurrence - one in a patient with severe shoulder pain who was found to have an osteolytic lesion and in two patients with lung nodules, one of whom had a history of breast cancer. One patient underwent Iodine-123 imaging prior to radioiodine ablation following surgery.

Following endogenous TSH stimulation after withdrawal of L-thyroxine therapy, all the patients had elevated TSH levels (TSH $>30 \mathrm{mIU} / \mathrm{l}$ ) before i.v. administration of Iodine-123. SPECT/CT of the neck and thorax was performed in all the patients as per our imaging protocol, and a further SPECT/CT of the abdomen/pelvis was performed in one patient, which revealed no abnormal radioiodine uptake. The reference standard was established by clinical follow-up including thyroglobulin levels in addition to histopathology $(n=5)$, neck ultrasound $(n=18)$, computed tomography $(n=17)$, gamma camera imaging of subsequent radioiodine therapy $(n=10)$, bone scintigraphy $(n=2)$, magnetic resonance imaging (MRI) of the neck $(n=2)$, MRI of the spine $(n=2)$, and 2-(18F)-fluoro-2-deoxyD-glucose positron emission tomography/computed tomography (FDG-PET/CT) $(n=2)$. Mean follow-up was 13 months.

\section{Diagnostic performance}

Based on the reference standard, there was evidence for residual or recurrent thyroid cancer in 22 out of 85 Iodine-123 imaging studies, 11 of which were non-radioiodine avid. In addition, nine studies had

Table 1 Patient characteristics.

\begin{tabular}{ll}
\hline Characteristics & Number $(n=79)$ \\
\hline $\begin{array}{l}\text { Participant age, years } \\
\text { Mean }\end{array}$ & 49 \\
Range & $16-85$ \\
Sex $n(\%)$ & \\
Female & $60(76 \%)$ \\
Male & $19(24 \%)$ \\
Histopathology $n(\%)$ & \\
Papillary thyroid cancer & $61(77 \%)$ \\
Follicular thyroid cancer & $17(22 \%)$ \\
Unknown & $1(1 \%)$ \\
Stage at diagnosis & 36 \\
I & 7 \\
II & 7 \\
III & 16 \\
IV & 13 \\
Unknown & \\
\hline
\end{tabular}


Table 2 Findings for whole body planar gamma camera (WBGC) imaging, with the addition of single photon emission computed tomography (SPECT) and SPECT/computed tomography (CT).

\begin{tabular}{|c|c|c|c|c|c|c|c|c|c|c|}
\hline \multirow[b]{2}{*}{ Modality } & \multicolumn{5}{|c|}{ Malignant $(n=22)$} & \multicolumn{5}{|c|}{ Benign $(n=63)$} \\
\hline & DA & PA & $E$ & PN & DN & DA & PA & $E$ & PN & DN \\
\hline WBGC imaging & 3 & 3 & 3 & 6 & 7 & 0 & 11 & 9 & 15 & 28 \\
\hline WBGC + SPECT imaging & 6 & 3 & 1 & 5 & 7 & 1 & 4 & 2 & 14 & 42 \\
\hline $\begin{array}{l}\text { WBGC + SPECT/CT } \\
\text { imaging }\end{array}$ & 10 & 1 & 0 & 0 & 11 & 0 & 0 & 0 & 8 & 55 \\
\hline
\end{tabular}

DA, definitely abnormal; PA, probably abnormal; E, equivocal; PN, probably normal; DN, definitely normal.

radioiodine-avid remnant thyroid tissue only; 54 studies had no evidence of disease. The diagnostic performance of WBGC imaging, SPECT, and SPECT/CT are presented in Tables 2 and 3. In a study-based analysis, SPECT/CT provided additional diagnostic information in $42 \%$ $(36 / 85)$ of cases.

The sensitivity was $41 \%$ for WBGC imaging, $45 \%$ with the addition of SPECT, and 50\% with the addition of SPECT/CT. However, although we observed an increase in sensitivity, this did not reach statistical significance for either WBGC imaging versus SPECT $(P=1.0)$ or WBGC imaging versus SPECT/CT $(P=0.5)$. When 11 cases who were found on follow-up to have non-radioiodine-avid disease were excluded, the sensitivity increased from $82 \%$ for WBGC imaging to $91 \%$ with the addition of SPECT and to $100 \%$ with the addition of SPECT/CT.

Nevertheless, there was a significant improvement in specificity from $68 \%$ for WBGC imaging to $89 \%$ $(P=0.0002)$ with the addition of SPECT. The addition of SPECT/CT further increased the specificity to $100 \%$ $(P<0.0001)$. The increase in specificity between SPECT and SPECT/CT also was statistically significant $(P=0.016)$.

A major factor in the increased specificity of SPECT and/or SPECT/CT was the reclassification of initially equivocal findings. There were 12 WBGC scans classed as equivocal, three remained so on SPECT, but none were on SPECT/CT. Classing equivocal findings as positive for disease, WBGC imaging was false-positive in 20 cases (nine thyroid remnants; four esophageal tracer retention; four asymmetric salivary glands; and three surface contamination) and false-negative in 13 cases (11 non-radioiodine-avid metastases and 2 cervical nodal metastases).

The addition of SPECT alone correctly classified 13 false-positive findings as physiological activity or remnant, and it identified metastatic neck lymph nodes in one study and additional bone metastases in another study. Therefore, the combination of WBGC and SPECT imaging produced 7 false-positive findings and 12 false-negative findings (Figs 1 and 2).

SPECT/CT correctly assigned all false-positive findings and identified a metastatic cervical lymph node misinterpreted as submandibular gland on WBGC and SPECT imaging alone. Several additional small bone metastases were identified on the CT component of the study in a patient already noted to have multiple bone metastases on WBGC and SPECT imaging. SPECT/CT was false-negative for 11 cases with non-radioiodineavid disease; five of these cases had metastatic neck lymph nodes, one with additional miliary lung metastases. One case had a $2.5 \mathrm{~cm}$ metastatic skin lesion on the neck which was subsequently excised, and three cases had small lung metastases on diagnostic CT which were not visible on the low-dose CT component of the SPECT/CT. In two cases, the sites of disease remain occult but were classed as radioiodine negative on the basis of a persistently elevated thyroglobulin.

\section{ROC analysis}

Following the exclusion of 11 cases found on follow-up to have non-radioiodine-avid disease, ROC curve analysis revealed an AUC of 0.84 for WBGC imaging, 0.95 for WBGC plus SPECT imaging, and 1.0 for WBGC plus SPECT/CT imaging (Fig. 3).

\section{Diagnostic confidence}

On a per study basis, overall diagnostic confidence increased with the addition of SPECT and SPECT/CT to WBGC imaging. The percentage of studies classed as 'definitely normal' or 'definitely abnormal' increased

Table 3 Diagnostic performance of whole body planar gamma camera (WBGC) imaging, with the addition of single photon emission computed tomography (SPECT) and SPECT/computed tomography $(\mathrm{CT})$.

\begin{tabular}{llll}
\hline & $\begin{array}{l}\text { WBGC } \\
\text { imaging }\end{array}$ & $\begin{array}{l}\text { WBGC+ } \\
\text { SPECT } \\
\text { imaging }\end{array}$ & $\begin{array}{l}\text { WBGC+ } \\
\text { SPECT/CT } \\
\text { imaging }\end{array}$ \\
\hline True-positive $(n)$ & 9 & 10 & 11 \\
False-positive $(n)$ & 20 & 7 & 0 \\
True-negative ( $n$ ) & 43 & 56 & 63 \\
False-negative ( $n$ ) & $13(2)$ & $12(1)$ & $11(0)$ \\
Sensitivity (\%) & $41(82)$ & $45(91)$ & $50(100)$ \\
Specificity (\%) & 68 & 89 & 100 \\
PPV (\%) & 31 & 59 & 100 \\
NPV (\%) & $77(96)$ & $82(98)$ & $85(100)$ \\
Accuracy (\%) & $61(70)$ & $78(89)$ & $87(100)$ \\
\hline
\end{tabular}

In the analysis, equivocal interpretation was considered malignant. Numbers in brackets refer to analysis of patients with radioiodine-avid disease only. 
(a)

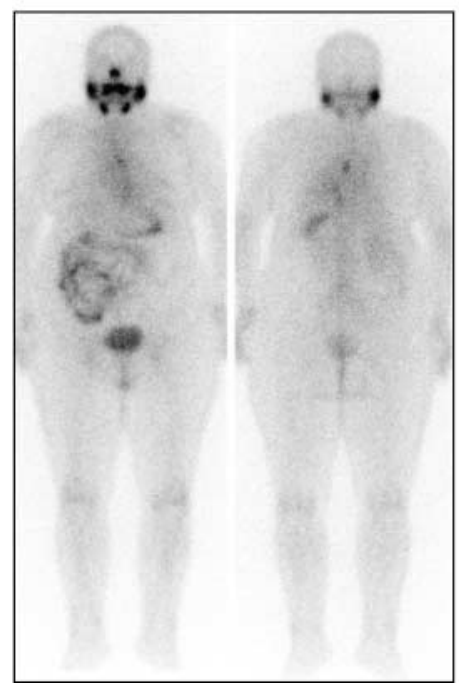

(b)

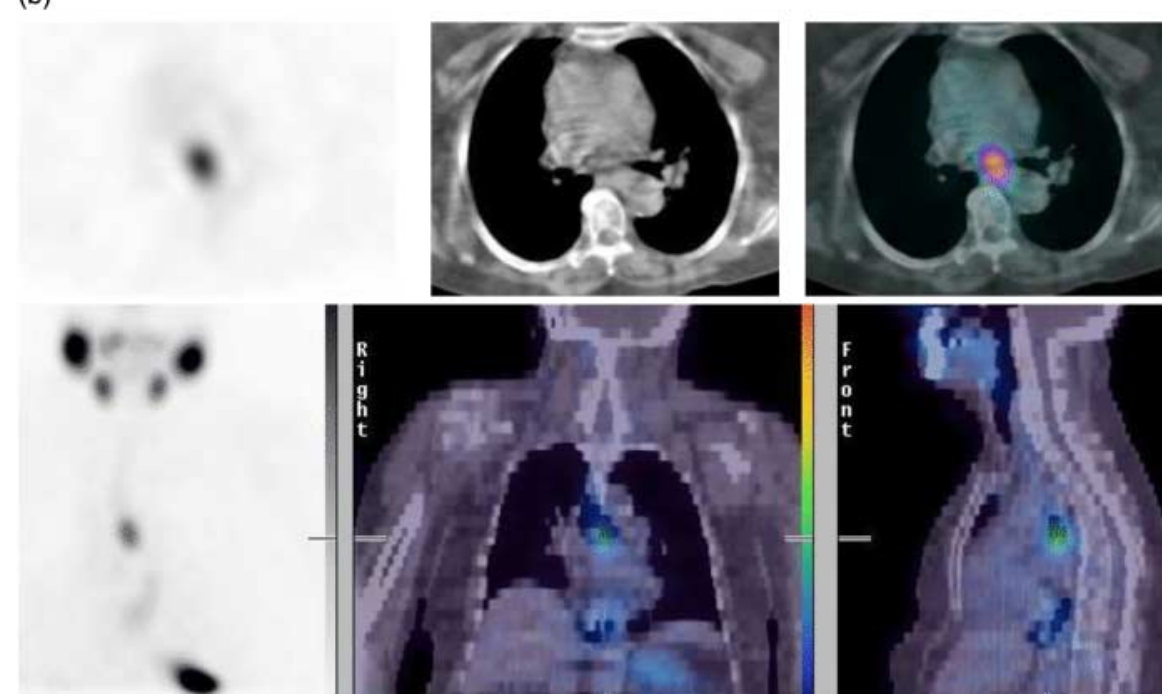

Figure 1 (a) Planar whole body lodine-123 gamma camera imaging in anterior and posterior views shows mildly increased linear activity in projection of the esophagus but more focal activity projected over the mediastinum. This was interpreted as equivocal for either radioiodine retention in the esophagus or a mediastinal lymph node metastasis. (b) The finding remained equivocal on SPECT but clearly localises to the esophagus on SPECT/CT. Full colour version of this figure available via http://dx.doi.org/10.1530/EJE-09-1023.

from $46 \%(39 / 85)$ with WBGC imaging to $66 \%(56 / 85)$ with the addition of SPECT to $89 \%(76 / 85)$ with the addition of SPECT/CT.

\section{Change in diagnostic findings}

The addition of SPECT/CT provided further characterization in $63(70 \%)$ of the 90 foci identified on 51 WBGC imaging studies (Table 4). A total of 41 foci initially thought to be either equivocal or pathological (false-positive) were reclassified by the addition of SPECT/CT as being thyroid remnant $(n=20)$ or other sites of physiological radioiodine uptake $(n=21)$. Five metastatic foci thought to be physiological (false-negative) were lymph node $(n=3)$ or bone metastases $(n=2)$. For 17 foci, the site of metastases was accurately localized.

\section{Change in patient management}

The addition of SPECT/CT to WBGC imaging led to a change in management in $9(11 \%)$ out of 85 cases. In two patients, identification of cervical lymph node metastases that were not clearly identified on WBGC imaging led to surgical lymph node dissection rather than radioiodine therapy. In one patient who was thought to have both cervical and mediastinal lymph node metastases, SPECT/CT localized the mediastinal activity to the esophagus. Therefore, the patient underwent a cervical lymph node dissection. In one patient who was thought to have localized cervical nodal disease, equivocal mediastinal uptake was shown to be a mediastinal lymph node, and the patient had radioiodine therapy rather than surgery. In five patients with elevated thyroglobulin levels, WBGC imaging was thought to be abnormal; however, SPECT/CT showed the increased Iodine-123 uptake to be physiological. These patients underwent diagnostic CT, neck ultrasound and FDG-PET/CT scans, and surgery or radiotherapy rather than radioiodine therapy.

In addition, in 11 cases who were thought to have abnormal Iodine-123 uptake on WBGC imaging, SPECT/CT did not confirm disease obviating the need for further investigations or avoiding ineffective radioiodine therapy. However, none of these patients had raised serum thyroglobulin levels, and most likely a 'watch-and-wait' policy would have been adopted. Thus, although SPECT/CT provided additional information in these cases, we did not anticipate a change in management.

\section{Discussion}

SPECT/CT improved the diagnostic performance of Iodine-123 imaging for differentiated thyroid cancer. The specificity increased from 68 to $89 \%$ with the addition of SPECT $(P=0.0002)$ and to $100 \%$ with the addition of SPECT/CT $(P<0.0001)$. The overall accuracy improved from $61 \%$ for planar gamma camera imaging to $78 \%$ with the addition of SPECT and to $87 \%$ with the addition of SPECT/CT. Furthermore, the diagnostic confidence of the observers improved such that we found no equivocal findings using SPECT/CT.

The accurate localization and characterization of focally increased Iodine-123 uptake is often not possible on planar gamma camera imaging, particularly in 
(a)

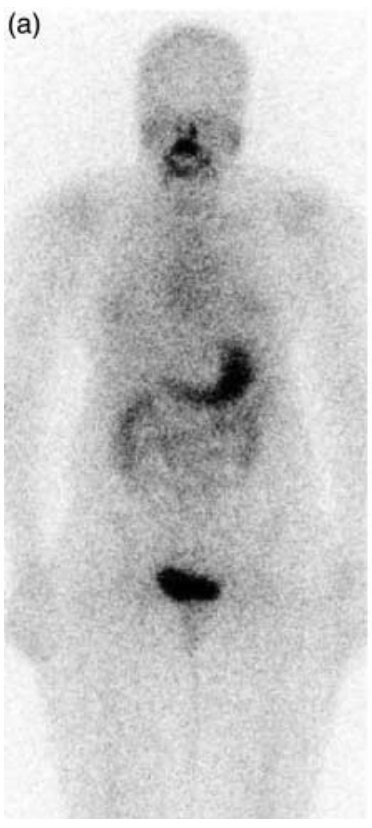

(b)
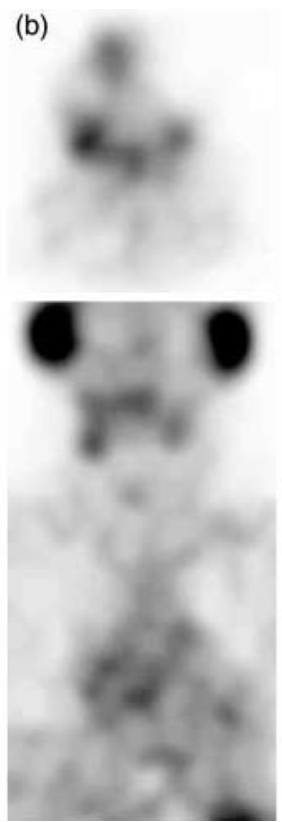
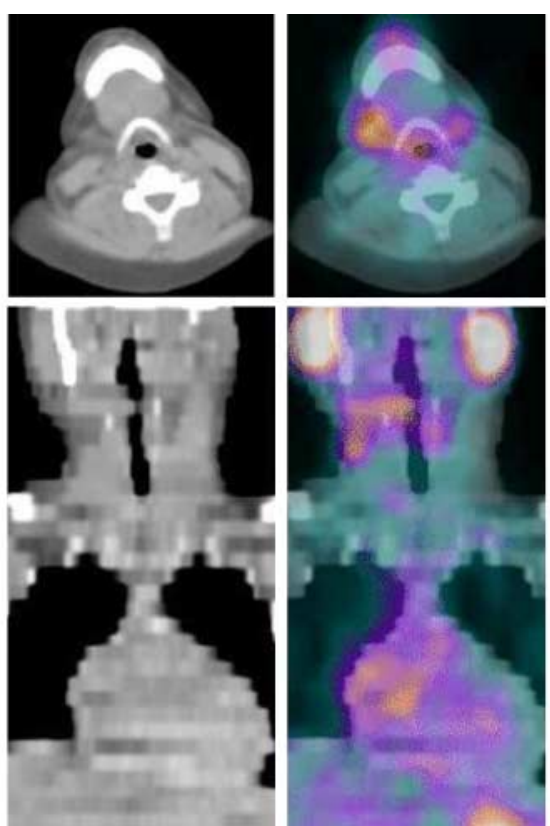

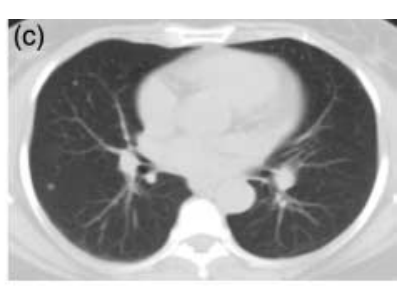

Figure 2 (a) Whole body planar lodine-123 gamma camera imaging in anterior view shows minor asymmetric uptake in the region of the submandibular glands thought to be probably normal. (b) SPECT/CT axial and coronal images: on SPECT there is discrete focal uptake bilaterally. On SPECT/CT this localised to several small bilateral cervical lymph nodes. (c) Axial diagnostic CT thorax (lung windows) demonstrates several small pulmonary nodules consistent with metastases, which were not apparent on SPECT. Full colour version of this figure available via http://dx.doi.org/10.1530/EJE-09-1023.

the neck and upper thorax. Although the addition of SPECT improved the diagnostic accuracy, it frequently remained difficult to differentiate between malignant and physiological radioiodine uptake. For example, we found it difficult to resolve between residual thyroid tissue and cervical lymph node metastases on whole body imaging with the addition of SPECT alone. Radioiodine retention in the esophagus was often recognized on planar imaging and SPECT by its typical linear configuration. However, focally increased activity is better characterized by SPECT/CT. In 13 studies, SPECT/CT enabled accurate localization of physiological radioiodine retention in the esophagus thought to be possibly pathological on planar gamma camera imaging and on SPECT. This is an important finding as in such cases the patient is spared further imaging procedures or even unnecessary radioiodine therapy (8).

The sensitivity of radioiodine whole body imaging depends on various factors including technical parameters, the activity administered and the reference standard used (12). Although we injected 350-400 MBq Iodine-123, the sensitivity was only $41 \%$ (9 out of 22), which is lower than 70-89\% sensitivity figures reported by others (13-15). However, previous studies used, for example, post-radioiodine therapy imaging as the reference standard; thus, all the patients had radioiodine-avid disease. In our study, follow-up revealed 11 cases with non-radioiodine-avid disease, which when excluded, resulted in a sensitivity of $82 \%$ for planar imaging, which compares well with these reports (13-15).

To date, there have been several studies addressing the use of radioiodine SPECT/CT in differentiated thyroid cancer (16-20). Fused SPECT/CT in 25 patients with inconclusive findings on post-radioiodine ablation imaging improved anatomical localization in 17 (44\%) out of 39 foci compared with SPECT alone (18). Furthermore, image interpretation changed in 15 $(38 \%)$ foci. In another study, SPECT/CT provided additional diagnostic information in 41 (57\%) out of 71 patients who underwent Iodine-131 imaging post radioiodine therapy $(n=54)$ or as a diagnostic procedure $(n=17)$ (19). SPECT/CT particularly improved the characterization and localization of increased Iodine-131 uptake in lymph node metastases, remnant thyroid tissue, lung and mediastinal as well as bone metastases. Similar results were found by others $(16,17,20)$. The additional diagnostic information of SPECT/CT over planar imaging ranged from 57 to $74 \%$ of patients $(16,17,19)$. When assessing the impact of SPECT/CT on patient management, we found a change in $11 \%$ of the patients. Previous reports found a change in 24 (17), 25 (18), and $41 \%$ of the patients (19). However, these studies were limited to patients with indeterminate findings on planar Iodine-131 imaging.

To the best of our knowledge, this is the first consecutive series using Iodine-123 SPECT/CT in the follow-up of thyroid cancer regardless of the findings on 


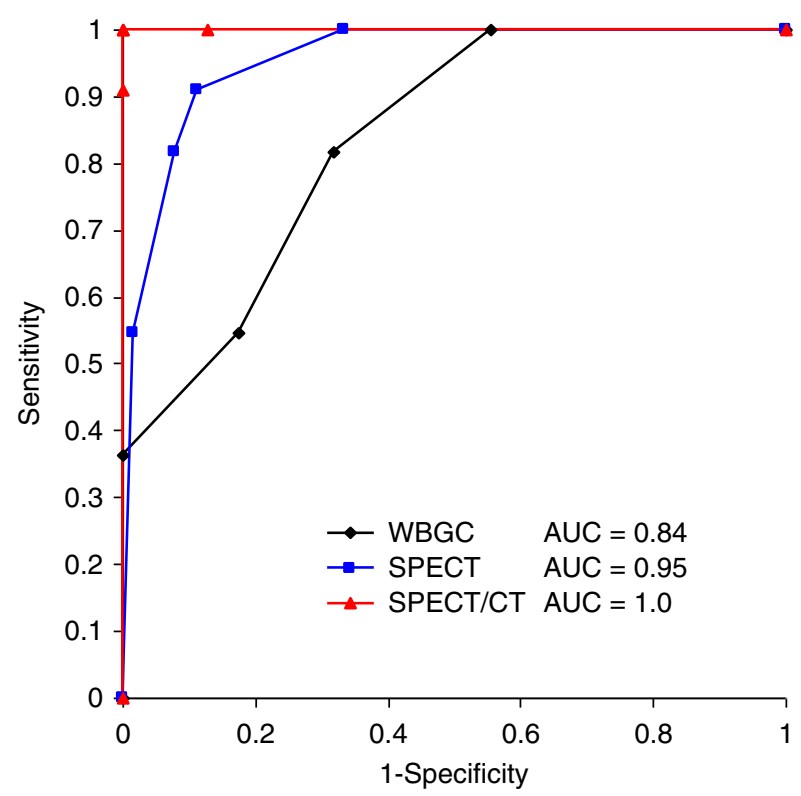

Figure 3 Receiver operating characteristic (ROC) curves of whole body gamma camera (WBGC) imaging versus WBGC + SPECT imaging versus WBGC+SPECT/CT imaging (patients with nonradioiodine-avid disease excluded). AUC, area under the curve. Ful colour version of this figure available via http://dx.doi.org/10.1530/ EJE-09-1023.

whole body imaging. However, previous studies have analyzed consecutive series of cases using Iodine131 imaging. Spanu et al. prospectively performed 117 SPECT/CT of the neck and thorax demonstrating 116 foci on planar imaging (21). SPECT/CT had incremental value over planar in $67.8 \%$ positive cases (40/59) and modified management in $35.6 \%$ positive cases $(21 / 59)$. This translated to an incremental value in 40/117 (34\%) studies and a change in management in $21 / 117(21 \%)$ studies. Of note, patients with non-radioiodine-avid disease were excluded from their analysis. Our findings compare well with additional diagnostic information of SPECT/CT in $42 \%$ of studies. Spanu et al. (21) found SPECT/CT to be particularly useful in revealing nodal metastases adjacent to salivary glands occult on planar imaging (4 cases in 3 patients). Similarly, we found three cases of cervical nodal metastases in two patients. These would be potentially missed in other studies which only perform SPECT/CT in equivocal findings on planar imaging. The specific advantage of SPECT/CT for nodal assessment of the neck is also emphasized by a recent study which found a gain in information on nodal stage in $20(35 \%)$ out of 57 patients leading to a change in risk stratification in $14(25 \%)$ patients (22).

We found a reduction in the number of equivocal/indeterminate findings with the addition of SPECT/CT, which is supported by a recent study where positive SPECT/CT predicted persistent/recurrent disease in $9(16 \%)$ out of 55 patients (23). Three out of nine patients $(33 \%)$ had non-radioiodine-avid disease and accounted for false-negative SPECT/CT as reported for our study (23).

In common with the majority of previous studies discussed above, the CT performed as part of SPECT/CT in our study did not contribute independent diagnostic information. We used a low resolution Hawkeye-CT, which was not of diagnostic image quality although the CT images provided valuable anatomical information regarding the precise localization of abnormal radioiodine uptake. More recently, SPECT/CT systems are incorporating diagnostic multislice $\mathrm{CT}$, capable of lowdose as well as diagnostic CT. Depending on the protocol used, these may permit even better lesion characterization and, as such, further reduce the need for subsequent diagnostic studies (24). It is also important

Table 4 Summary of the additional characterization of whole body gamma camera (WBGC) findings provided by single photon emission computed tomography (SPECT)/CT in 63 out of 90 foci.

\begin{tabular}{|c|c|c|c|c|c|}
\hline WBGC findings & $\begin{array}{l}\text { Number } \\
\text { of foci }\end{array}$ & Characterization by SPECT/CT & $\begin{array}{l}\text { Number } \\
\text { of foci }\end{array}$ & Additional value & $\begin{array}{l}\text { Number } \\
\text { of foci }\end{array}$ \\
\hline \multirow[t]{2}{*}{$\begin{array}{l}\text { Not seen on planar GC } \\
\text { imaging }\end{array}$} & \multirow[t]{2}{*}{4} & Bone metastases & 2 & \multirow[t]{3}{*}{$\begin{array}{l}\text { Metastatic foci not seen on } \\
\text { planar GC imaging }\end{array}$} & \multirow[t]{3}{*}{5} \\
\hline & & Cervical lymph node metastases & 2 & & \\
\hline $\begin{array}{l}\text { Probably salivary gland } \\
\text { uptake }\end{array}$ & 1 & Cervical lymph node metastasis & 1 & & \\
\hline Abnormal cervical activity & 5 & Cervical lymph node metastases & 5 & \multirow[t]{3}{*}{ Localization of metastases } & \multirow[t]{4}{*}{17} \\
\hline \multirow[t]{2}{*}{ Abnormal thoracic activity } & 10 & Bone metastases & 4 & & \\
\hline & & $\begin{array}{l}\text { Mediastinal lymph node } \\
\text { metastases }\end{array}$ & 6 & & \\
\hline Abnormal pelvic activity & 2 & Bone metastases & 2 & & \\
\hline \multirow[t]{4}{*}{ Abnormal cervical activity } & 35 & $\begin{array}{l}\text { Physiological uptake in } \\
\text { submandibular gland }\end{array}$ & 4 & \multirow[t]{6}{*}{ Exclusion of disease } & \multirow[t]{6}{*}{41} \\
\hline & & Physiological esophageal activity & 9 & & \\
\hline & & Contamination & 2 & & \\
\hline & & Thyroid bed remnant & 20 & & \\
\hline Abnormal thoracic activity & 5 & Physiological esophageal activity & 5 & & \\
\hline Abnormal shoulder activity & 1 & Contamination & 1 & & \\
\hline
\end{tabular}


to consider the additional radiation exposure from the CT portion of SPECT/CT, which in our cases was $\sim 1 \mathrm{mSv}$ in addition to $\sim 5 \mathrm{mSv}$ from Iodine-123 imaging.

There are some limitations of this study which need to be considered. The diagnostic performance was patientbased. This may underestimate the fraction of 'falsenegative' findings. For example, two patients had lung metastases which were negative on SPECT/CT, but the patients were considered 'true-positive' for the purpose of a patient-based analysis as cervical metastases were correctly identified. This is a general limitation as a detailed focus based analysis is challenging because histopathologic confirmation would be difficult as it is not feasible to sample all potential sites of disease.

The number of patients with radioiodine-avid disease was limited. Patients subsequently shown to have nonradioiodine-avid disease were retained in this analysis of consecutive patients. This resulted in lower sensitivities compared with previous reports. However, the primary purpose of the study was to assess the incremental value of adding SPECT and SPECT/CT to conventional planar gamma camera imaging. This analysis is not biased by the study population. For the same reason, thyroglobulin levels or findings from neck ultrasound or any other clinical information were not made available to the image reviewers.

In conclusion, our study has shown that the addition of SPECT/CT improved the diagnostic performance of Iodine-123 whole body planar imaging and SPECT alone. With the increasing availability of latest generation SPECT/CT systems, implementation into routine clinical radioiodine imaging should be considered. Future studies should assess the optimum protocol, impact on patient outcome, and cost-effectiveness.

\section{Declaration of interest}

The authors declare that there is no conflict of interest that could be perceived as prejudicing the impartiality of the research reported.

\section{Funding}

This research did not receive any specific grant from any funding agency in the public, commercial, or not-for-profit sector. Only internal departmental resources were used.

\section{Acknowledgements}

We would like to thank Mr Tri Tat from the Department of Statistics, Imperial College, London, UK, for statistical support.

\section{References}

1 DeGroot LJ, Kaplan EL, McCormick M \& Straus FH. Natural history, treatment, and course of papillary thyroid carcinoma. Journal of Clinical Endocrinology and Metabolism 199071 414-424.

2 Hay ID, Bergstralh EJ, Goellner JR, Ebersold JR \& Grant CS. Predicting outcome in papillary thyroid carcinoma: development of a reliable prognostic scoring system in a cohort of 1779 patients surgically treated at one institution during 1940 through 1989. Surgery 1993114 1050-1057.

3 Hundahl SA, Fleming ID, Fremgen AM \& Menck HR. A National Cancer Data Base report on 53,856 cases of thyroid carcinoma treated in the U.S., 1985-1995. Cancer 199883 2638-2648.

4 Mazzaferri EL \& Jhiang SM. Long-term impact of initial surgical and medical therapy on papillary and follicular thyroid cancer. American Journal of Medicine 199497 418-428.

5 Siddiqi A, Foley RR, Britton KE, Sibtain A, Plowman PN, Grossman AB, Monson JP \& Besser GM. The role of ${ }^{123}$ I-diagnostic imaging in the follow-up of patients with differentiated thyroid carcinoma as compared to ${ }^{131}$ I-scanning: avoidance of negative therapeutic uptake due to stunning. Clinical Endocrinology 2001 55 515-521.

6 Carlisle MR, Lu C \& McDougall IR. The interpretation of ${ }^{131}$ I scans in the evaluation of thyroid cancer, with an emphasis on false positive findings. Nuclear Medicine Communications 200324 715-735.

7 Mitchell G, Pratt BE, Vini L, McCready VR \& Harmer CL. False positive ${ }^{131}$ I whole body scans in thyroid cancer. British Journal of Radiology 200073 627-635.

8 Leitha T \& Staudenherz A. Frequency of diagnostic dilemmas in ${ }^{131}$ I whole body scanning. Nuklearmedizin $20034255-62$.

9 Seo Y, Mari C \& Hasegawa BH. Technological development and advances in single-photon emission computed tomography/ computed tomography. Seminars in Nuclear Medicine 200838 177-198.

10 Townsend DW \& Cherry SR. Combining anatomy and function: the path to true image fusion. European Journal of Radiology 2001 11 1968-1974.

11 Hawass NE. Comparing the sensitivities and specificities of two diagnostic procedures performed on the same group of patients. British Journal of Radiology $1997 \mathbf{7 0}$ 360-366.

12 Lubin E, Mechlis-Frish S, Zatz S, Shimoni A, Segal K, Avraham A, Levy R \& Feinmesser R. Serum thyroglobulin and iodine-131 whole-body scan in the diagnosis and assessment of treatment for metastatic differentiated thyroid carcinoma. Journal of Nuclear Medicine 199435 257-262.

13 de Geus-Oei LF, Oei HY, Hennemann G \& Krenning EP. Sensitivity of ${ }^{123} \mathrm{I}$ whole-body scan and thyroglobulin in the detection of metastases or recurrent differentiated thyroid cancer. European Journal of Nuclear Medicine and Molecular Imaging 200229 768-774.

14 Gerard SK \& Cavalieri RR. I-123 diagnostic thyroid tumor wholebody scanning with imaging at 6,24 , and 48 hours. Clinical Nuclear Medicine 200227 1-8.

15 Park HM, Park YH \& Zhou XH. Detection of thyroid remnant/metastasis without stunning: an ongoing dilemma. Thyroid 19977 $277-280$.

16 Chen L, Luo Q, Shen Y, Yu Y, Yuan Z, Lu H \& Zhu R. Incremental value of ${ }^{131}$ I SPECT/CT in the management of patients with differentiated thyroid carcinoma. Journal of Nuclear Medicine 2008 49 1952-1957.

17 Kohlfuerst S, Igerc I, Lobnig M, Gallowitsch HJ, Gomez-Segovia I, Matschnig S, Mayr J, Mikosch P, Beheshti M \& Lind P. Posttherapeutic (131)I SPECT-CT offers high diagnostic accuracy when the findings on conventional planar imaging are inconclusive and allows a tailored patient treatment regimen. European Journal of Nuclear Medicine and Molecular Imaging 200936 886-893.

18 Ruf J, Lehmkuhl L, Bertram H, Sandrock D, Amthauer H, Humplik B, Ludwig Munz D \& Felix R. Impact of SPECT and integrated low-dose CT after radioiodine therapy on the management of patients with thyroid carcinoma. Nuclear Medicine Communications 200425 1177-1182.

19 Tharp K, Israel O, Hausmann J, Bettman L, Martin WH, Daitzchman M, Sandler MP \& Delbeke D. Impact of ${ }^{131}$ I-SPECT/CT 
images obtained with an integrated system in the follow-up of patients with thyroid carcinoma. European Journal of Nuclear Medicine and Molecular Imaging 200431 1435-1442.

20 Wong KK, Zarzhevsky N, Cahill JM, Frey KA \& Avram AM. Incremental value of diagnostic ${ }^{131}$ I SPECT/CT fusion imaging in the evaluation of differentiated thyroid carcinoma. American Journal of Roentgenology 2008191 1785-1794.

21 Spanu A, Solinas ME, Chessa F, Sanna D, Nuvoli S \& Madeddu G. ${ }^{131}$ I SPECT/CT, in the follow-up of differentiated thyroid carcinoma: incremental value versus planar imaging. Journal of Nuclear Medicine 200950 184-190.

22 Schmidt D, Szikszai A, Linke R, Bautz W \& Kuwert T. Impact of ${ }^{131} \mathrm{I}$ SPECT/spiral $\mathrm{CT}$ on nodal staging of differentiated thyroid carcinoma at the first radioablation. Journal of Nuclear Medicine 200950 18-23.
23 Aide N, Heutte N, Rame JP, Rousseau E, Loiseau C, Henry-Amar M \& Bardet S. Clinical relevance of single-photon emission computed tomography/computed tomography of the neck and thorax in postablation (131)I scintigraphy for thyroid cancer. Journal of Clinical Endocrinology and Metabolism $2009 \mathbf{9 4}$ 2075-2084.

24 Buck AK, Nekolla S, Ziegler S, Beer A, Krause BJ, Herrmann K, Scheidhauer K, Wester HJ, Rummeny EJ, Schwaiger M \& Drzezga A. SPECT/CT. Journal of Nuclear Medicine $2008 \mathbf{4 9}$ 1305-1319.

Received 12 February 2010

Accepted 8 March 2010 